\title{
Geometry-Based Finite-Element Modeling of the Electrical Contact Between a Cultured Neuron and a Microelectrode
}

\author{
Jan Reinoud Buitenweg*, Wim L. C. Rutten, and Enrico Marani
}

\begin{abstract}
The electrical contact between a substrate embedded microelectrode and a cultured neuron depends on the geometry of the neuron-electrode interface. Interpretation and improvement of these contacts requires proper modeling of all coupling mechanisms. In literature, it is common practice to model the neuron-electrode contact using lumped circuits in which large simplifications are made in the representation of the interface geometry. In this paper, the finite-element method is used to model the neuron-electrode interface, which permits numerical solutions for a variety of interface geometries. The simulation results offer detailed spatial and temporal information about the combined electrical behavior of extracellular volume, electrode-electrolyte interface and neuronal membrane.
\end{abstract}

Index Terms-Ccultured neurons, finite-element modeling, multielectrode arrays, neuron-electrode contact.

\section{INTRODUCTION}

$\mathbf{P}$ LANAR substrate microelectrode arrays (MEAs) offer the possibility to contact neuronal cultures with a large number of cell-size electrodes. They are explored as a tool for long term extracellular recording from and stimulation of cultured neuronal networks [1]-[5] and for the development of a cultured neuron probe, a new type of neuro-electronic interface [6]-[8].

So far, a diversity of shapes and a wide range of amplitudes of extracellularly recorded action potentials from neuronal cultures on MEAs have been reported [9]-[12], indicating variability in the behavior of the electrical contacts between the neurons and the microelectrodes. These contacts appear to depend on geometry, as amplitudes of recorded action potentials increase with electrode coverage and with the resistance of the sealing gap between the neuronal membrane and the substrate.

Manuscript received August 24, 2001; revised November 5, 2002. This work was part of the NESTING project (Neuro-Electronic Systems toward Tissue Implantation by Neural Grafting) and was supported by the BIOMED II EC project under shared-cost Contract BMH4-2723. Asterisk indicates corresponding author.

*J. R. Buitenweg is with the Institute for Biomedical Technology, Signals \& Systems Group, Faculty of Electrical Engineering, University of Twente, P.O. Box 217, 7500 AE Enschede, The Netherlands (e-mail: J.R.Buitenweg@el.utwente.nl).

W. L. C. Rutten are with the Institute for Biomedical Technology, Signals \& Systems Group, Faculty of Electrical Engineering, University of Twente, 7500 AE Enschede, The Netherlands.

E. Marani is with the Institute for Biomedical Technology, Signals \& Systems Group, Faculty of Electrical Engineering, University of Twente, 7500 AE Enschede, The Netherlands, and the Neuroregulation Group, Department of Neurosurgery, Leiden University Medical Centre, 2300 RC Leiden, The Netherlands.

Digital Object Identifier 10.1109/TBME.2003.809486

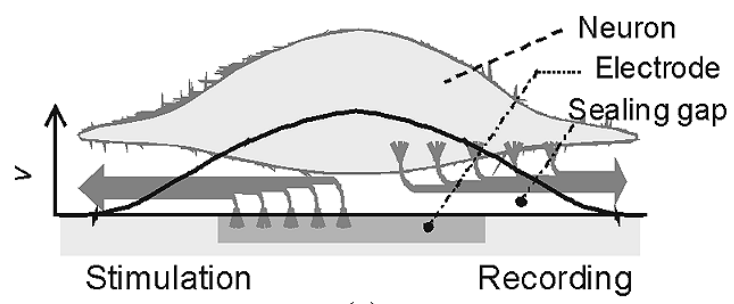

(a)

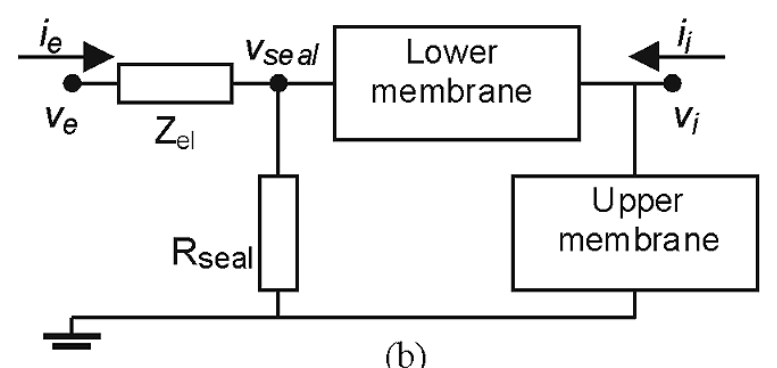

Fig. 1. (a) Mediation of the neuron-electrode interface in extracellular stimulation and recording. Due to current densities, arising from the neuronal membrane or the electrode, a potential distribution exists in the sealing gap which modifies the membrane potential (stimulation) or can be probed by the electrode (recording). (b) Electrical equivalent lumped circuit which is commonly used as a model of the neuron-electrode contact.

Evaluation and improvement of the neuron-electrode contact requires understanding of the coupling mechanisms involved, which are 1) volume conduction through the extracellular space, 2) electrical interaction of the volume conductor with the surface of the electrode, and 3) with the neuronal membrane. This volume conductor and its electrical interactions with the electrode and the neuron is the primary subject of this paper and it is called the neuron-electrode interface [Fig. 1(a)].

Understanding of the combined action of these coupling mechanisms depends on proper geometry-based modeling of the neuron electrode interface. When a neuron covers, or seals, an electrode completely, the electrical behavior of the interface is primarily determined by the sealing gap, which can be represented mathematically by the "core-coat conductor" equations, as proposed by Weis and Fromherz [13]. These equations describe a two-dimensional resistive sheet, which interacts with the neuronal membrane and with the silicon substrate, including the gate of a field effect transistor. An analytical solution of these equations can be found for simple, e.g., axisymmetrical sealing gaps, but not for more complex geometries. Therefore, it has become common practice to model the neuron-electrode 


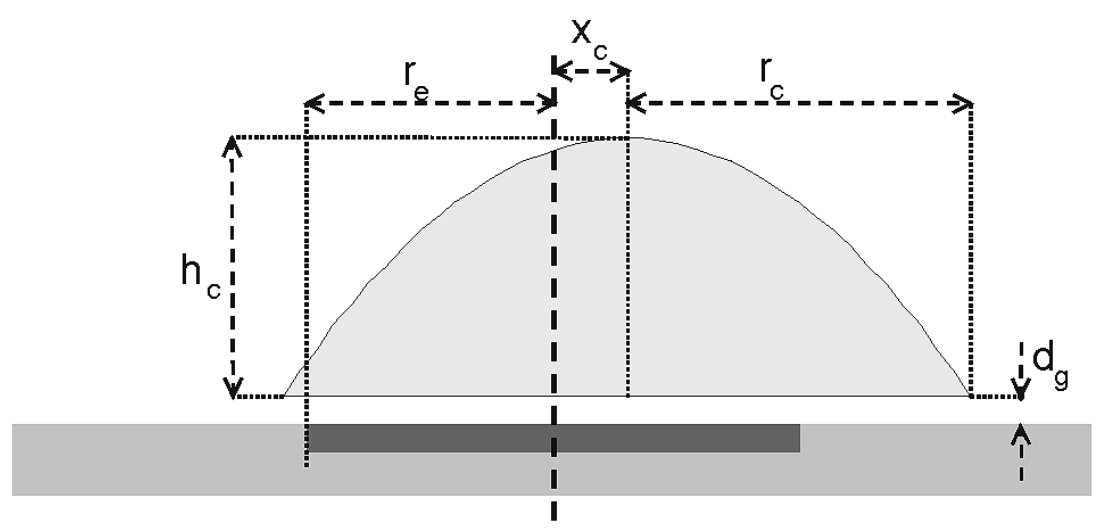

Fig. 2. Parametrical geometry of the neuron-electrode interface.

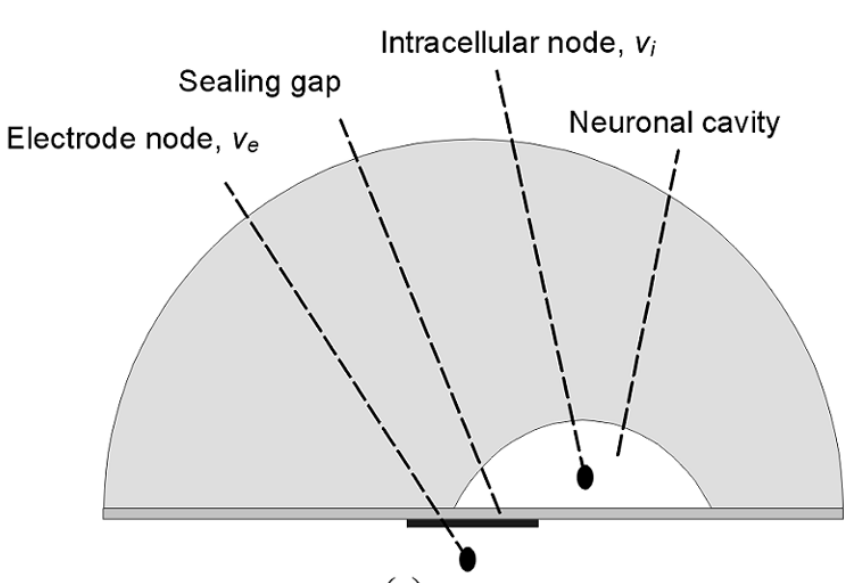

(a)

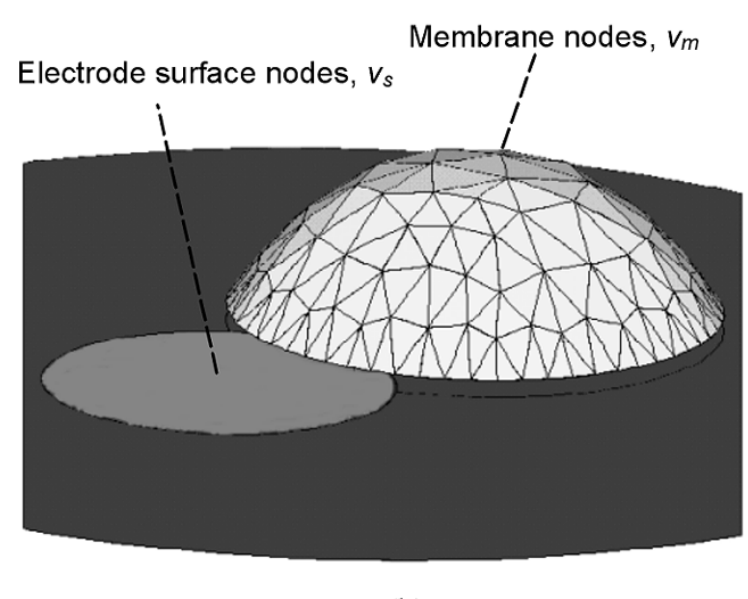

(b)

Fig. 3. (a) Implementation of the volume conductor geometry in the ANSYS finite-element software. (b) Three-dimensional visualization of the neuron electrode interface geometry in case of defect sealing.

contact with simple lumped circuits of which the parameters are based on a rule of translation of the core-coat conductor model and/or experimental data [10], [13]-[16]. In these circuits, the membrane is split up into an upper part and a lower part over the sealing gap which can be defined as the free membrane and the junction membrane, respectively [Fig. 1(b)]. Furthermore, the electrode-electrolyte interface and the sealing resistance are represented by single components.

Although lumped circuit models (LCM) have been used with some success, it is not guaranteed that these models offer a geometry related understanding of the neuron-electrode contact for at least two reasons. First, large simplifications are made in the representation of the electrical properties of the interface by the definition of only four components. Second, it is difficult to compute the model parameters from the geometry, unless the geometry is very simple (e.g., axisymmetrical).

In this paper, a finite-element model (FEM) is proposed as a tool for studying the electrical properties of the neuron-electrode interface. This model permits numerical solution of volume conductor problems for a variety of geometries, including partial and complete sealing. The spatial and temporal interaction of the sealing gap with the neuronal membrane and the electrode-electrolyte interface is included in the model. Although active membrane properties (voltage-gated channels) can be implemented easily, only passive membrane properties will be considered in this paper. Simulations are focused on the above-mentioned coupling mechanisms and on the influence of the interface geometry in cases of intracellular and extracellular stimulation.

\section{METHODS}

\section{A. Parametrical Geometry}

A parametrical representation of the geometry of the neuronelectrode interface will be used (Fig. 2). The neuron is modeled as a circular soma of radius $r_{c}$, with a parabolic height $\left(h_{c}\right)$ profile. The neuron is positioned with an eccentricity $x_{c}$ on top of an electrode with radius $r_{e}$. With increasing eccentricity, the sealing remains complete until $x_{c}<\left(r_{c}-r_{e}\right)$ and becomes defect when $x_{c}>\left(r_{c}-r_{e}\right)$. A sealing gap of thickness $d_{g}$ is modeled between the soma and the substrate or the electrode.

\section{B. FEM}

The geometry of the volume conductor surrounding the interface consists of the culture medium around the neuron and in the sealing gap and is implemented in ANSYS Finite Element Software (SAS IP Inc. Houston) release 5.4 (Fig. 3). The medium around the neuron is modeled as the upper half of a sphere (radius of $40 \mu \mathrm{m}$ ) from which the neuronal volume is removed. 
The neuronal cavity is closed by connection of a volume layer of thickness $d_{g}$ below this sphere, resulting in a sealing gap below the neuron. Variation of the location of the neuronal cavity permits modeling of complete as well as defect sealing.

Both volumes are meshed and filled with tetrahedral shaped volume elements (SOLID98 and SOLID5) which permit numerical solution of the Poisson equation

$$
\vec{\nabla} \cdot\left(\sigma_{\text {medium }} \vec{\nabla} v\right)=0
$$

with $v$ the electrical potential in the volume and $\sigma_{\text {medium }}=$ $1.65 \mathrm{~S} / \mathrm{m}$ the conductivity of the culture medium. The size of the elements varied from $0.5 \mu \mathrm{m}$ in the sealing gap to several microns far away from the neuron-electrode interface. The nodes at the outer boundary of the modeled part of the culture medium are set to zero potential, representing a counter electrode, which is far away from the neuron-electrode interface (Dirichlet conditions).

The relationship between extracellular potentials and membrane and electrode current densities is obtained from ANSYS. The total volume conductor problem is solved partially, resulting in a relationship containing only the nodes at the neuronal membrane and at the electrode surface

$$
K_{m s} \cdot\left[\begin{array}{c}
\vec{v}_{m} \\
\vec{v}_{s}
\end{array}\right]=\left[\begin{array}{l}
\vec{i}_{m} \\
\vec{i}_{s}
\end{array}\right]
$$

with vectors $\vec{v}_{m}$ and $\vec{v}_{s}$ representing the extracellular potential at the membrane nodes and the potential at the nodes of the electrode surface, respectively. The vectors $\vec{i}_{m}$ and $\vec{i}_{s}$ represent the currents into these nodes. The indexes of the conductivity matrix $K_{m s}$, as computed in ANSYS, refer to the indexes of the potential and current vectors.

The conductivity matrix $K_{m s}$ is transferred to the MATLAB environment for further use. The interaction of the volume conductor with the neuronal membrane and the electrode surface are obtained by addition of an intracellular node and an electrode node. Subsequently, the membrane and electrode surface potentials are redefined with respect to the intracellular and electrode potentials, respectively

$$
\left[\begin{array}{cc}
K_{m s}^{\prime} & K_{m s i e} \\
K_{m s i e}^{T} & K_{i e}
\end{array}\right] \cdot\left[\begin{array}{c}
\vec{v}_{m}^{\prime} \\
\vec{v}_{s}^{\prime} \\
v_{i} \\
v_{e}
\end{array}\right]=\left[\begin{array}{c}
\vec{i}_{m} \\
\vec{i}_{s} \\
i_{i} \\
i_{e}
\end{array}\right]
$$

with $v_{i}$ the intracellular potential, $v_{e}$ the electrode potential

$$
\vec{v}_{m}^{\prime}=\left[\begin{array}{c}
v_{i}-v_{m, 1} \\
\vdots \\
v_{i}-v_{m, N_{m}}
\end{array}\right]
$$

the local transmembrane potentials (with $N_{m}$ the number of membrane nodes), and

$$
\vec{v}_{s}^{\prime}=\left[\begin{array}{c}
v_{e}-v_{s, 1} \\
\vdots \\
v_{e}-v_{s, N_{s}}
\end{array}\right]
$$

the local trans-electrode-electrolyte potentials (with $N_{s}$ the number of electrode nodes). The local outward membrane currents $\vec{i}_{m}$ and the local electrode surface currents $\vec{i}_{s}$ can now be expressed for each node as a function of the transmembrane potential, the trans-electrode-electrolyte potential, and the intracellularly or extracellularly injected stimulation currents, $i_{i}$ and $i_{e}$, respectively

$$
\begin{aligned}
& {\left[\begin{array}{l}
\vec{i}_{m} \\
\vec{i}_{s}
\end{array}\right]=K_{m s i e} \cdot K_{i e}^{-1} \cdot\left[\begin{array}{l}
i_{i} \\
i_{e}
\end{array}\right]} \\
& \quad+\left(K_{m s}^{\prime}-K_{m s i e} \cdot K_{i e}^{-1} \cdot K_{m s i e}^{T}\right) \cdot\left[\begin{array}{c}
\vec{v}_{m}^{\prime} \\
\vec{v}_{s}^{\prime}
\end{array}\right] .
\end{aligned}
$$

Assuming a passive membrane, the derivative of the local membrane potentials can be computed for each node $n=1 \cdots N_{m}$

$$
\frac{d}{d t} v_{m, n}^{\prime}=\frac{i_{m, n}-A_{m, n} \cdot g_{m} \cdot\left(v_{m, n}^{\prime}-v_{r m p}\right)}{A_{m, n} \cdot c_{m}}
$$

with $A_{m, n}$ the membrane area represented by node $n, g_{m}=0.3$ $\mathrm{mS} / \mathrm{cm}^{2}$ the local membrane conductivity, and $c_{m}=1 \mu \mathrm{F} / \mathrm{cm}^{2}$ the membrane capacity per unit area. The resting membrane potential is defined as $v_{\mathrm{rmp}}=-65 \mathrm{mV}$.

For a platinum electrode, the electrode-electrolyte interface is assumed to have only a capacitive impedance. Therefore, the derivative of the local trans-electrode-electrolyte potentials is computed for each node $n=1 \cdots N_{s}$ as

$$
\frac{d}{d t} v_{s, n}^{\prime}=\frac{i_{s, n}}{A_{s, n} \cdot c_{e}}
$$

with $A_{s, n}$ the electrode area represented by electrode node $n$ and $c_{e}=500 \mu \mathrm{F} / \mathrm{cm}^{2}$ the electrode capacity per unit area. The value of $c_{e}$ is based on the impedance of a platinized electrode of $10-\mu \mathrm{m}$ diameter at $1 \mathrm{kHz}$, which is around $400 \mathrm{k} \Omega$.

The total set of partial difference equations [(6)-(8)] is solved numerically in MATLAB version 5.3 (The Mathworks, Inc., Natick, MA), using a variable order solver, based on numerical differentiation formulas, (ODE15s) with a maximum timestep of $10 \mu \mathrm{s}$. The initial conditions at $t=0$ were taken $v_{m, n}^{\prime}=$ $v_{\text {rmp }}\left(n=1 \cdots N_{m}\right)$ and $v_{s, n}^{\prime}=0\left(n=1 \cdots N_{s}\right)$. After solving the partial differential equations, the intracellular potential and the potential at the electrode, can be computed for all time instants from (3) as

$$
\left[\begin{array}{c}
v_{i} \\
v_{e}
\end{array}\right]=K_{i e}^{-1} \cdot\left[\begin{array}{c}
i_{i} \\
i_{e}
\end{array}\right]+K_{i e}^{-1} \cdot K_{m s i e}^{T} \cdot\left[\begin{array}{c}
\vec{v}_{m}^{\prime} \\
\vec{v}_{s}^{\prime}
\end{array}\right] .
$$

An overview of symbols for potentials and currents is given in Table I.

\section{Simulations}

In Table II, the parameters of all modeled geometries are summarized. A variety of neuronal sizes is found in different parts of the nervous system. Even within a population of a specific type of neurons, larger and smaller subtypes are often distinguished. Therefore, the radius of the neuron is varied from $r_{c}=7$ to $r_{c}=20 \mu \mathrm{m}$ in four steps. These values are representative for a wide variety of vertebral neuronal types, such as cortical neurons, dorsal root ganglion neurons, and spinal cord neurons [17]-[19]. The height of the neuron varies linearly with its radius from $h_{c}=5 \mu \mathrm{m}$ to $h_{c}=14 \mu \mathrm{m}\left(h_{c} \approx 0.7 \cdot r_{c}\right)$, based on the assumption of a certain flattening of the neuron in culture, due to adhesion to the substrate. 
TABLE I

List OF SYMBOLS USED FOR POTENTIALS AND CURRENTS

\begin{tabular}{c|l}
\hline Symbol & Description \\
\hline$v_{i}$ & Intracellular potential \\
$v_{e}$ & Electrode potential (in the metal) \\
$\vec{v}_{m}$. & Extracellular potential at the membrane nodes \\
$\vec{v}_{m}$ & Local transmembrane potentials \\
$\vec{v}_{s}$ & Extracellular potential at the electrode nodes \\
$\vec{v}_{s}$ & Local trans-electrode-electrolyte potentials \\
$i_{i}$ & Intracellular stimulation current \\
$i_{e}$ & Extracellular stimulation current \\
$\vec{i}_{m}$ & Local outward membrane currents \\
$\vec{i}_{s}$ & Local outward electrode surface currents \\
\hline
\end{tabular}

TABLE II

LIST OF GEOMETRIES USED FOR SIMULATIONS

\begin{tabular}{c|ccccc}
\hline $\begin{array}{c}\text { Geometry } \\
\text { No. }\end{array}$ & $\begin{array}{c}x_{c} \\
{[\mu \mathrm{m}]}\end{array}$ & $\begin{array}{c}r_{c} \\
{[\mu \mathrm{m}]}\end{array}$ & $\begin{array}{c}h_{c} \\
{[\mu \mathrm{m}]}\end{array}$ & $\begin{array}{c}r_{e} \\
{[\mu \mathrm{m}]}\end{array}$ & $\begin{array}{c}d_{g} \\
{[\mathrm{~nm}]}\end{array}$ \\
\hline 1 & 0 & 7 & 5 & 5 & 10 \\
2 & 0 & 7 & 5 & 5 & 50 \\
3 & 0 & 7 & 5 & 5 & 100 \\
4 & 0 & 10 & 7 & 5 & 50 \\
5 & 0 & 15 & 10 & 3 & 50 \\
6 & 0 & 15 & 10 & 5 & 50 \\
7 & 0 & 15 & 10 & 10 & 50 \\
8 & 0 & 20 & 15 & 5 & 50 \\
9 & 12 & 20 & 15 & 5 & 50 \\
10 & 18 & 20 & 15 & 5 & 50 \\
\hline
\end{tabular}

The value of the electrode radius, $r_{e}=5 \mu \mathrm{m}$, is based on the design of the MEA that is currently in use in our group [20]. Since the size of the electrode is a major design parameter, the electrode radius is varied from $r_{e}=3 \mu \mathrm{m}$ to $r_{e}=10 \mu \mathrm{m}$. For the thickness of the sealing gap, values of $d_{g}=10,50$, and $100 \mathrm{~nm}$ are taken, representing a range from strong to weak neuron-substrate adhesion [21], [22].

Since cultured neurons rarely cover an electrode completely, the effects of varying eccentricity of the neuron are studied using a number of eccentricities in the range from complete sealing to defect sealing.

Simulation of extracellular stimulation is performed by application of a 2-ms current pulse of $1 \mathrm{nA}$ through the extracellular electrode node. Intracellular stimulation is simulated by injection of a 2-ms current pulse of $100 \mathrm{pA}$ into the intracellular node.

\section{RESULTS}

\section{A. Complete and Defect Sealing}

Geometries 8-10 (see Table II) were used for simulation of the effect of eccentricity on the neuron-electrode contact using FEM (Fig. 4). Intracellular current stimulation with 100 pA results in a transient depolarization of the intracellular potential during the stimulation pulse. Furthermore, a potential distribution builds up in the sealing gap. Both the intracellular potential and the potential distribution in the sealing gap are not affected by variation of the geometry. However, the computed electrode potential decreases with the eccentricity of the neuron. This extracellular response to the stimulus is still recorded when the sealing has become defect. Amplitude and shape of the response appear to be proportional to the stimulation pulse, although a transient is observed which becomes more dominant when the eccentricity of the neuron increases.

In case of extracellular stimulation, the potential distribution in the sealing gap builds up due to the current densities arising from the electrode surface. As a consequence, this distribution is now governed by the position of the neuron. The potential distribution reaches a maximum above the surface of the electrode, but also extends to the sealing gap around it. The intracellular response decreases with the eccentricity of the neuron. Even at defect sealing, there is still a noticeable effect of the stimulation current. The amplitude and shape of the intracellular potential are proportional to the stimulation pulse, although a transient is observed which again increases with the eccentricity. The extracellular response also depends on the geometry, but only during the stimulus pulse: after termination of the stimulus, the electrode potential remains at a constant value. During the stimulus, the response can be described as an almost rectangular pulse, decreasing with the eccentricity of the neuron, which is superimposed on an invariable ramp toward the post stimulus value.

\section{B. Interactions in the Sealing Gap}

The mediating role of the sealing gap in the interaction between the neuronal membrane and the electrode is studied in more detail. For this purpose, the lower membrane potential is presented as a function of the distance, $r$, to the center of the electrode for a symmetrical geometry $\left(x_{c}=0\right)$. At a distance $r=r_{c}$, the lower membrane meets the upper membrane, over which the potential is uniformly distributed (Fig. 4). Hence, this radial presentation with $r=0 \cdots r_{c}$, represents the total membrane potential distribution.

In Fig. 5, these radial distributions are presented at several time instants during the simulation (geometry 1 ). At $t=0.5 \mathrm{~ms}$, no stimulus is present and the membrane potential equals $v_{\text {rmp. }}$. At $t=1 \mathrm{~ms}$, an extracellular stimulation current $(1 \mathrm{nA})$ is applied resulting in a potential distribution which is flat and hyperpolarized over the electrode area $\left(r_{e}=5 \mu \mathrm{m}\right)$, but makes a transition to a slightly depolarized upper membrane potential, somewhere in the sealing gap. During the stimulation pulse, the initially flat part of the potential distribution changes to a more "parabolic" shape, which sustains for a short period after termination of the stimulation pulse.

The intracellular response to the stimulus is depicted in the inset of Fig. 5 and the time instants of the radial distributions, presented above, are marked in the largest trace. The changes in these distributions, during and immediately after the stimulus, are accompanied by a transient in the intracellular response. Both the amplitude and the transient of this response are affected by variations of the thickness of the sealing gap, $d_{g}$, as can be seen from the other traces in the inset.

\section{Size of Neuron and Electrode}

Similar radial distributions of the membrane potential and the potential in the sealing gap are presented in Fig. 6 for variations in the size of the electrode and the neuron. Smaller electrodes 

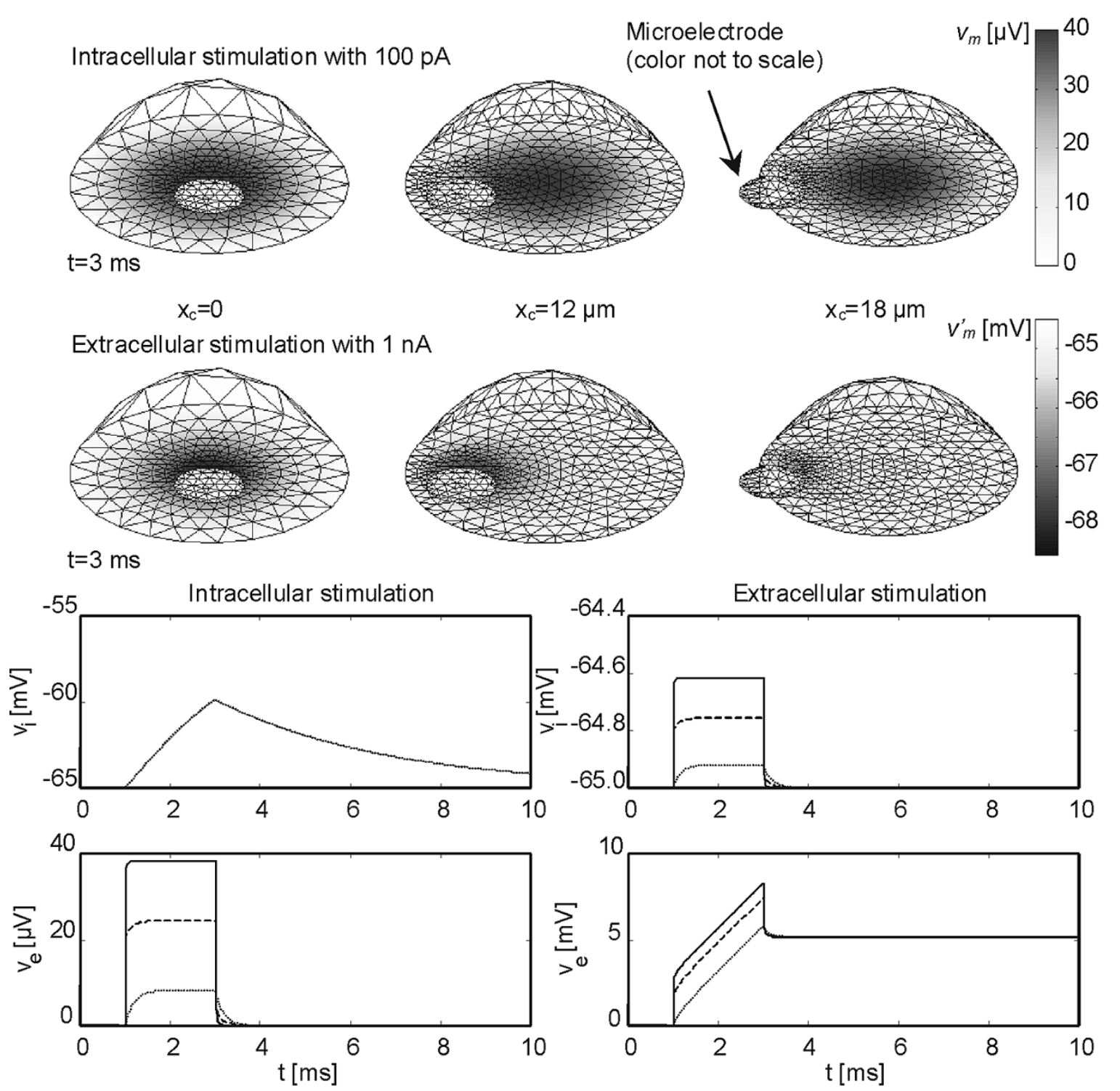

Fig. 4. FEM simulation of intracellular and extracellular stimulation with several geometries, varying from complete to defect sealing $\left(x_{c}=0, x_{c}=12\right.$, and $x_{c}=18 \mu \mathrm{m}$; see Table II, geometries 8, 9, and 10, respectively). Rectangular anodic stimulus pulses of $2 \mathrm{~ms}$ were applied at $t=1 \mathrm{~ms}$. In the top row, the extracellular potential distribution, $v_{m}$, around the neuronal membrane at $t=3 \mathrm{~ms}$ due to an intracellular current pulse of $100 \mathrm{pA}$ are plotted. In the next row, the distribution of local transmembrane potentials, $v_{m}^{\prime}$, at $t=3 \mathrm{~ms}$, due to an extracellular current pulse of $1 \mathrm{nA}$ is plotted. For the geometries above, the intracellular potential and the electrode potential are plotted versus time for both intracellular and extracellular stimulation (solid line: $x_{c}=0$, dashed line: $x_{c}=12 \mu \mathrm{m}$, and dotted line: $\left.x_{c}=18 \mu \mathrm{m}\right)$.

and larger neurons have a positive effect on the intracellular responses to extracellular stimuli [Fig. 6(a), (c)]. These responses are governed by the upper membrane potential. However, the lower membrane is affected much more than the upper membrane potential, as can be seen also in Figs. 4 and 5 .

Intracellular stimulation results in a potential distribution in the sealing gap which depends on the neuronal radius, but does not depend on the size of the electrode [Fig. 6(b), (d)]. As in the case of varying eccentricity, however (Fig. 4), the extracellularly recorded responses vary due to the applied differences in the geometry: Smaller electrodes and larger neurons produce recordings with higher amplitudes.

The transients in all responses (insets), which were also observed in the previous figures, are affected by the size of the electrode and the neuron: Larger electrodes [Fig. 6(a), (b)] and smaller neurons [Fig. 6(c), (d)] result in more dominant transients.

\section{DISCUSSION}

\section{A. Coupling Mechanisms}

An FEM is presented in this paper, to offer a geometry related insight into the combined action of the three coupling mechanisms involved in the neuron-electrode contact. These mechanisms, already mentioned in the introduction, are addressed below, together with the influence of the geometry as suggested by FEM.

Volume Conduction Through the Extracellular Space: The thin gap below the lower membrane plays a major role in the 


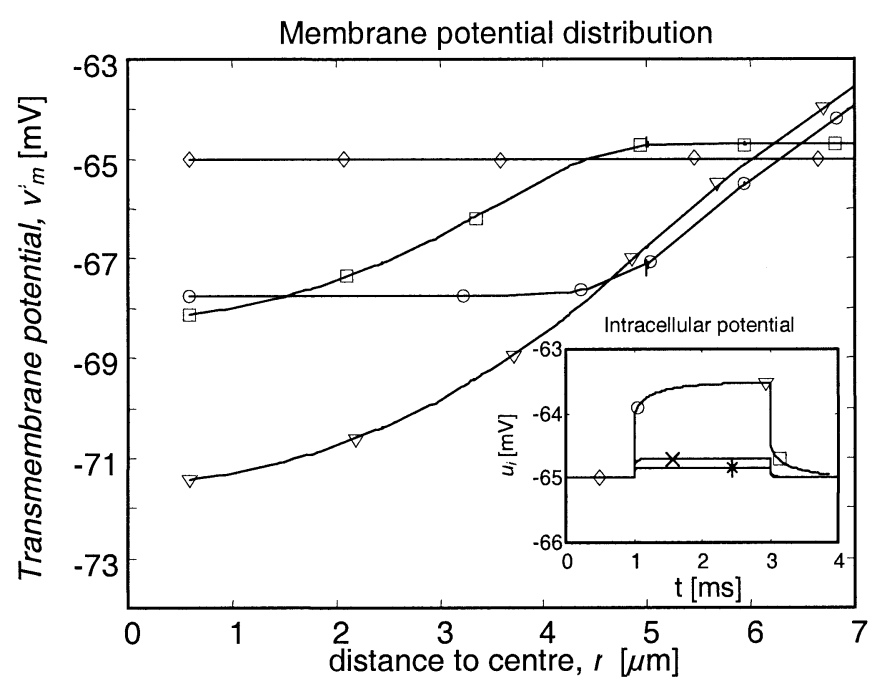

Fig. 5. Development of the lower membrane potential distribution for geometry $1\left(d_{g}=10 \mathrm{~nm}\right)$ due to an extracellular stimulation pulse. The lower membrane potential is plotted versus the distance to the center of the neuron-electrode interface at the time instances marked in the inset. Inset: the response of the intracellular potential. Also, the responses for $d g=50 \mathrm{~nm}$ (" $\times$ ") and $d g=100 \mathrm{~nm}$ ("*") are plotted (geometries 2 and 3, respectively, see Table II)

electrical contact, as also recognized by other investigators. Current densities through this sealing gap, arising from the electrode surface (extracellular stimulation) or from the neuronal membrane (intracellular stimulation), induce potentials which influence the local transmembrane potentials or the potential at the electrode (Fig. 4). In addition, the results from FEM suggest that the potential distribution not only builds up in the sealing gap around the electrode, but also in the gap over the electrode surface (Fig. 6).

Electrical Interaction Between the Volume Conductor and the Electrode Surface: Since the potential distribution varies over the electrode surface, the local trans-electrode-electrolyte potentials must be adjusted in order to match with the potential in the electrode metal. Due to the capacitive nature of the electrode, this adjustment requires local current densities through the electrode surface. In case of intracellular stimulation, no current is applied to the extracellular electrode, so the total charge on the electrode-electrolyte interface is not altered. Hence, the local trans-electrode-electrolyte potentials are adjusted by rearranging the local charges using currents from the sealing gap, until the electrode metal potential equals the potential distribution in the sealing gap, averaged over the electrode surface (assumed that no charge was present before the onset of the stimulus). The required currents for this averaging effect, give rise to a second effect of the interaction: the leakage of high frequency components of the current densities in the sealing gap. The most simple way to explain this effect is by considering intracellular stimulation and a geometry of partial electrode coverage, such as geometry $10\left(x_{c}=18 \mu \mathrm{m}\right.$, Fig. 4). Due to the averaging effect, currents will enter the electrode from the sealing gap and leave the electrode through the uncovered surface of the electrode. These currents escape from the sealing gap and will not contribute to the potential distribution above the electrode, until the averaging is completed. Hence, the observed transients in the extracellular response to intracellular stimulation can be explained by the settling of an equilibrium between the potentials in the sealing gap and the trans-electrode-electrolyte potentials. In case of complete sealing, an additional potential builds up over the sealing gap around the electrode. This potential contributes to the potential over the electrode, resulting in a less dominant transient in the extracellular response. In case of extracellular stimulation, the averaging and high-frequency leakage effects also occur, resulting in the observed transients in the intracellular response. The transient development of the transmembrane potential, due to the potential distribution in the sealing gap is clearly illustrated in Fig. 5. The time constant of this transient depends on the thickness of the sealing gap and on the capacity of the electrode: A thinner sealing gap and higher electrode capacity will increase the timeconstant of the transient. In addition to the averaging effect, the electrode-electrolyte interface is charged by the extracellular stimulation current. After termination of this current, this charge remains, resulting in the observed post-stimulus potential at the electrode.

Electrical Interaction Between the Volume Conductor and the Neuronal Membrane: Similar interactions, as described above, also hold for interaction with the neuronal membrane. Extracellular stimulation results in depolarized and hyperpolarized regions of the membrane (Figs. 4 and 6). However, the membrane capacity $\left(c_{m}=1 \mu \mathrm{F} / \mathrm{cm}^{2}\right)$ is very small compared with the electrode capacity $\left(c_{e}=500 \mu \mathrm{F} / \mathrm{cm}^{2}\right)$, so the time-constant of the transients is to small to be recognized on this timescale.

\section{B. Geometry-Based Modeling}

In literature, several models of the electrical contact between neurons and microtransducers have been presented. From a geometrical point of view, the "core-coat conductor" model, including the spatial distribution of potentials and currents in the sealing gap [13], is the most detailed model of such an interface, so far proposed in literature. The FEM presented in this paper is based on a similar approach, although some important differences exists. First of all, this FEM is modeling a neuronelectrode interface instead of a neuron-transistor interface. Therefore, the capacitive coupling with the conducting silicon substrate is not implemented, but instead a (far more) capacitive electrode-electrolyte interface is modeled. Of course, the metal part of this interface is not connected to zero potential and the electrode area does not exist over the entire substrate. Second, the volume conducting part of the model includes the entire space around the neuron, not just the sealing gap, which permits consideration of a continuum of geometries, varying from complete to defect sealing. Finally, the solution is obtained numerically, and is not restricted to the simple (i.e., axisymmetrical) geometries required for analytical solutions.

A more simple and practical approach for understanding the relationship between intracellular and extracellular potentials and currents, is the use of LCM. A frequently used LCM is the "point contact" model [Fig. 1(b)] [10], [13]-[16]. The major difference between this model and FEM is the number of elements between the intracellular and the extracellular nodes, which has consequences for the representation of the geometry and the above-mentioned coupling mechanisms. The volume conduction through the extracellular space is represented by 


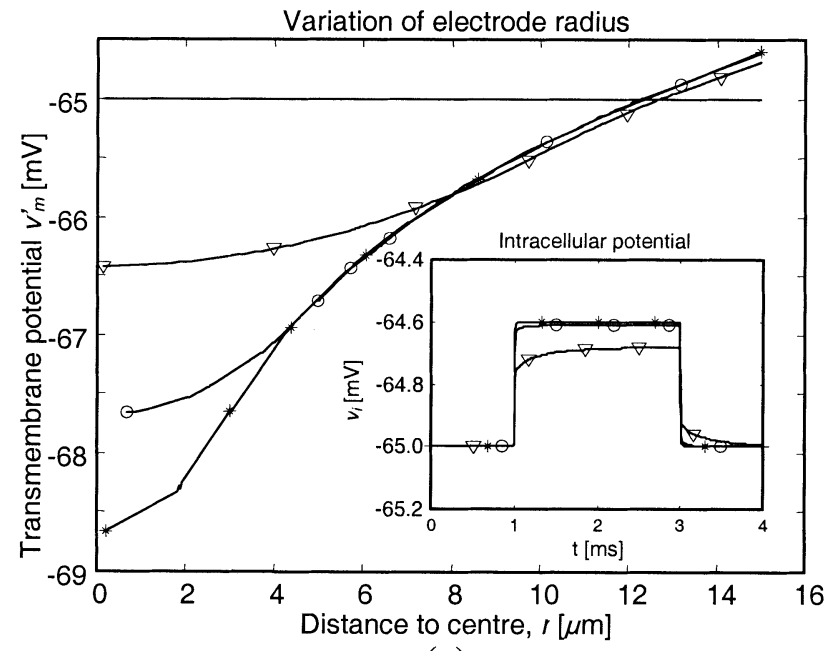

(a)

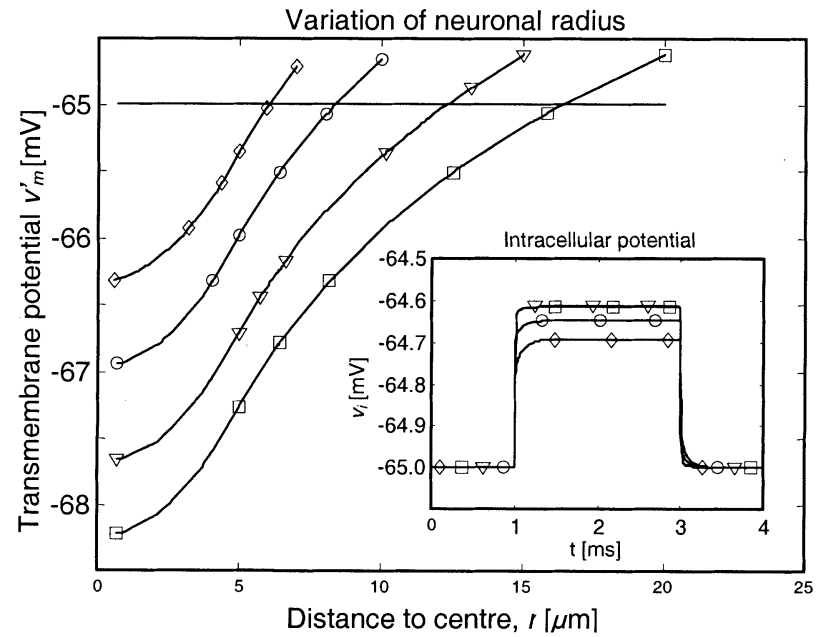

(c)

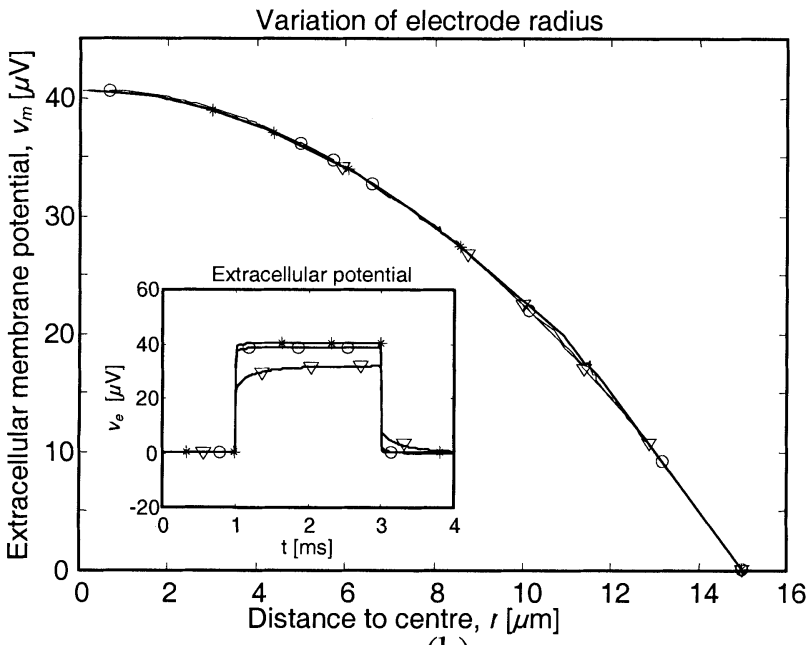

(b)

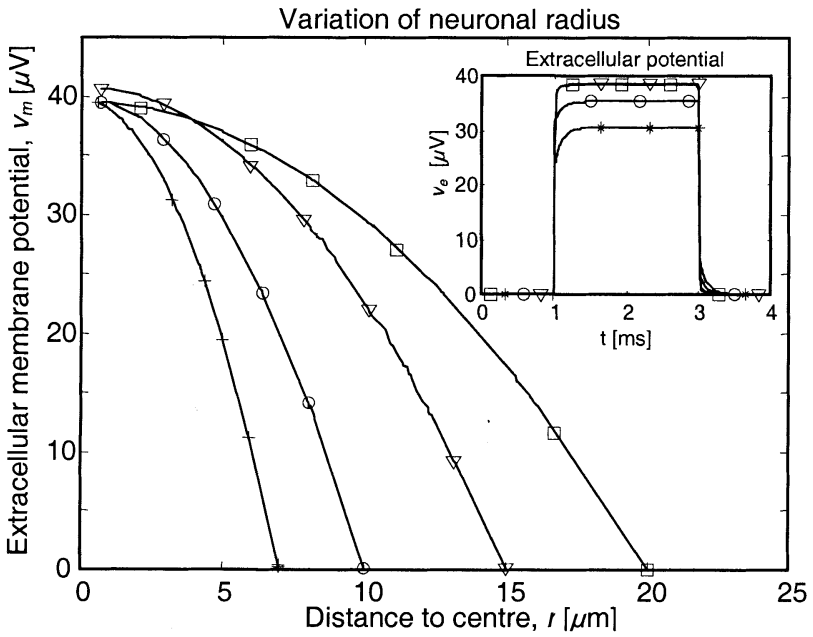

(d)

Fig. 6. Simulated effect of electrode radius and neuronal radius on the neuron-electrode contact. The potential of the lower membrane (a,c) and in the sealing gap [(b), (d)] are plotted versus the distance to the center of the neuron $\left(x_{c}=0\right)$ at $t=2.9$ ms for extracellular and intracellular stimulation, respectively. [(a), (b)] Simulations with electrode radius $r_{e}=3 \mu \mathrm{m}(*), r_{e}=5 \mu \mathrm{m}(\mathrm{o})$ and $r_{e}=10 \mu \mathrm{m}(\nabla)$ (geometries 5-7). [(c), (d)] Simulations with neuronal radius $r_{c}=7 \mu \mathrm{m}$ $(\diamond), r_{c}=10 \mu \mathrm{m}(\mathrm{o}), r_{c}=15 \mu \mathrm{m}(\nabla)$ and $r_{c}=20 \mu \mathrm{m}$ ( ) (geometries 2, 4, 6, and 8). Insets: Intracellular [(a), (c)] and electrode potential [(b), (d)] versus time.

a single sealing resistance, although different current distributions exist in the sealing gap for intracellular and extracellular stimulation (Fig. 4). Furthermore, since the potential distribution in the sealing gap is represented by a single potential, the modeled interaction between the volume conductor and the electrode will not include the averaging and high frequency leakage effect, so the observed transients in the responses [Fig. 6 (insets)] will not be produced by LCM. For modeling the interaction of the volume conductor with the neuronal membrane, the membrane is divided into an upper and a lower part. Hence, the membrane potential distribution is represented by two potentials. Besides on local membrane properties, these two membrane components should be based on the depolarized or hyperpolarized areas of the neuronal surface. However, the regions suggested by FEM do not correspond directly to the geometrically defined upper and lower membrane (Fig. 2), since the lower membrane can be partly hyperpolarized and depolarized at the same time [Fig. 6(a), (c)]. Furthermore, different areas are depolarized or hyperpolarized in case of intracellular and extracellular stimulation, especially in cases of defect sealing (Fig. 4).

So, although the LCM approach is simple and practical, a geometry-based selection or interpretation of the LCM parameters is difficult. However, if LCM parameters are properly selected, the responses predicted by both models should correspond after settling of the transients predicted by FEM, i.e., in steady-state. When the properties of the neuronal membrane are passive (no voltage sensitive channels), as assumed in this paper, the LCM steady-state response of the extracellular potential to an intracellular stimulation current (i.e., no current through the electrode) can be formulated as

$$
v_{e}^{(\text {intra })}=\alpha^{(\text {intra })} \cdot R_{\text {seal }} \cdot i_{i}
$$

with $R_{\text {seal }}$ the resistance of the sealing gap [see Fig. 1(b)] and $\alpha^{\text {(intra) }}$ the fraction of the applied stimulation current, $i_{i}$, that flows through the lower membrane into the sealing gap (note that the superscript "intra" refers to intracellular stimulation, 
TABLE III

Extraction of Passive Contact Parameters From FEM Results

\begin{tabular}{|c|c|c|c|c|c|c|}
\hline \multirow{2}{*}{$\begin{array}{c}\text { Geometry } \\
\text { No. }\end{array}$} & \multicolumn{4}{|c|}{$\begin{array}{l}\text { Extracellular stimulation } \\
\text { with } i_{e}=1 \mathrm{nA}\end{array}$} & \multicolumn{2}{|c|}{$\begin{array}{c}\text { Intracellular stimulation } \\
\text { with } i_{i}=100 \mathrm{pA}\end{array}$} \\
\hline & $\begin{array}{c}v_{\text {seal }}^{(\mathrm{extra})} \\
{[\mathrm{mV}]}\end{array}$ & $\begin{array}{c}\Delta v_{i}^{\text {(extra) }} \\
{[\mathrm{mV}]}\end{array}$ & $\begin{array}{c}R_{\text {seal }} \\
\text { eq.(15) } \\
{[\mathrm{M} \Omega]}\end{array}$ & $\begin{array}{l}\alpha^{\text {(extra) }} \\
\text { eq. (16) }\end{array}$ & $\begin{array}{c}v_{e}^{\text {(intra) }} \\
{[\mu \mathrm{V}]}\end{array}$ & $\begin{array}{l}\alpha^{(\text {intra) }} \\
\text { eq.(13) }\end{array}$ \\
\hline 1 & 5.60 & 1.48 & 5.60 & 0.263 & 146.07 & 0.261 \\
\hline 2 & 1.14 & 0.31 & 1.14 & 0.268 & 30.46 & 0.266 \\
\hline 3 & 0.58 & 0.16 & 0.58 & 0.273 & 15.90 & 0.273 \\
\hline 4 & 1.81 & 0.35 & 1.81 & 0.195 & 35.28 & 0.195 \\
\hline 5 & 3.50 & 0.40 & 3.50 & 0.114 & 40.03 & 0.114 \\
\hline 6 & 2.57 & 0.39 & 2.57 & 0.151 & 38.73 & 0.151 \\
\hline 7 & 1.25 & 0.32 & 1.25 & 0.253 & 31.80 & 0.253 \\
\hline 8 & 3.12 & 0.38 & 3.12 & 0.123 & 38.36 & 0.123 \\
\hline 9 & 2.29 & 0.25 & 2.29 & 0.107 & 24.55 & 0.107 \\
\hline 10 & 0.64 & 0.08 & 0.64 & 0.128 & 8.20 & 0.129 \\
\hline
\end{tabular}

not intracellular potential). According to LCM, an extracellular current stimulus will cause a potential in the sealing gap of

$$
v_{\text {seal }}^{(\text {extra })}=v_{e}^{(\text {extra })}-v_{e l}^{(\text {extra })}=R_{\text {seal }} \cdot i_{e}
$$

this potential cannot be measured in experimental practice, but can be derived by subtracting the potential over the electrodeelectrolyte interface, $v_{e l}$, from the measured extracellular potential $v_{e}$. The intracellular response, i.e., the change in the intracellular potential, can be formulated as a fraction, $\alpha^{(\text {extra })}$, of the potential in the sealing gap

$$
\Delta v_{i}^{(\text {extra })}=\alpha^{(\text {extra })} \cdot v_{\text {seal }}^{(\text {extra })}=\alpha^{(\text {extra })} \cdot R_{\text {seal }} \cdot i_{e} .
$$

According to these equations, the passive steady-state responses to intracellular or extracellular stimulation, depend on the product of two parameters: the sealing resistance, $R_{\text {seal }}$, and a fractional constant, $\alpha^{\text {(intra) }}$ or $\alpha^{\text {(extra) }}$. In Table III, these passive contact parameters are extracted using the FEM results and (10)-(12). The potential in the sealing gap, $v_{\text {seal }}^{(\text {extra })}$, required for calculation of the sealing resistance, is obtained by subtracting the post-stimulus value of $v_{e}$ from the maximum of the response, just before termination of the extracellular stimulus current (Fig. 4). The results in Table III demonstrate that for each modeled geometry, passive contact parameters can be identified for which the LCM and FEM steady-state responses correspond. The extracted values suggest that sealing resistance is not entirely descriptive for the neuron-electrode contact: in some cases a higher sealing resistance does not imply an improved intracellular or extracellular response (cfg. geometries 4 and 9). Hence, for interpreting a measured sealing resistance, information about $\alpha^{\text {(intra) }}$ and $\alpha^{\text {(extra) }}$ should also be available. The extracted values for $\alpha^{\text {(intra) }}$ and $\alpha^{\text {(extra) }}$ appear to be identical, apart from small numerical errors. Since the membrane conductivity is uniformly distributed and the resistance of the lower membrane area is much higher than the sealing resistance, these fractional constants can be interpreted as the fraction of the total membrane area involved in the contact.

As a conclusion it can be stated that the presented FEM offers detailed spatial and temporal information about the in- fluence of the interface geometry on the combined electrical behavior of extracellular volume, electrode-electrolyte interface and neuronal membrane. Although identification of passive contact parameters for use with LCM is possible, the relationship between these parameters and the (parametrical) interface geometry is rather complex. Of course, the consequences of the coupling mechanisms for extracellular recording and stimulation of bioelectrical activity requires modeling of active membrane properties (i.e., voltage-gated channels). These properties can be implemented easily by reformulation of (7), which is addressed in other papers [24], [25].

\section{ACKNOWLEDGMENT}

The authors would like to thank K. Peter and M. Deenen for their excellent support of the culturing activities in their laboratory and M. Goedbloed for the fabrication of the MEAs used in the experiments.

\section{REFERENCES}

[1] Y. Jimbo and A. Kawana, "Electrical stimulation and recording from cultured neurons using a planar electrode array," Bioelectrochem. Bioeng., vol. 29, pp. 193-204, 1992.

[2] Y. Jimbo, H. P. Robinson, and A. Kawana, "Strengthening of synchronized activity by tetanic stimulation in cortical cultures: Application of planar electrode arrays," IEEE Trans. Biomed. Eng., vol. 45, pp. 1297-1304, Nov. 1998.

[3] G. W. Gross, B. K. Rhoades, D. L. Reust, and F. U. Schwalm, "Stimulation of monolayer networks in culture through thin-film indium-tin oxide recording electrodes," J. Neurosc. Meth., vol. 50, pp. 131-143, 1993.

[4] J. van Pelt, P. Wolters, D. van Veen, J. Bomer, W. L. C. Rutten, H. Overdijk, and G. J. A. Ramakers, "Long-term multielectrode registration of neuronal firing activity from rat cerebral cortex tissue in vitro," in Proc. Int. Conf. IEEE Eng. Med. \& Biol. Soc., Amsterdam, The Netherlands, 1996, ISBN 90-9 010 005-9 (CDROM).

[5] M. P. Maher, J. Pine, J. Wright, and Y. C. Tai, "The neurochip: A new multielectrode device for stimulating and recording from cultured neurons," J. Neurosc. Meth., vol. 87, pp. 45-56, 1999.

[6] J. Pine, M. Maher, S. Potter, Y.-C. Tai, S. Tatic-Lucic, and J. Wright, "A cultured neuron probe," in Proc. Int. Conf. IEEE Engineering in Medicine and Biology Soc., Amsterdam, 1996, ISBN 90-9010 005-9 (CDROM).

[7] W. L. C. Rutten, "Neurotechnology," in Wiley Encyclopedia of Electrical and Electronics Engineering, J. G. Webster, Ed. New York: Wiley, 1999, vol. 14, pp. 366-373. 
[8] W. L. C. Rutten, J. P. A. Smit, T. A. Frieswijk, J. A. Bielen, A. L. H Brouwer, J. R. Buitenweg, and T. Heida, "Neuro-electronic interfacing with multielectrode arrays," IEEE Eng. Med. Biol. Mag., vol. 18, pp. $47-55,1999$.

[9] G. W. Gross, "Simultaneous single unit recording in vitro with a photoetched laser deinsulated gold multimicroelectrode surface," IEEE Trans. Biomed. Eng., vol. BME-26, pp. 273-279, 1979.

[10] W. G. Regehr, J. Pine, C. S. Cohan, M. D. Mischke, and D. W. Tank, "Sealing cultured invertebrate neurons to embedded dish electrodes facilitates long-term stimulation and recording," J. Neurosc. Meth., vol. 30, pp. 91-106, 1989.

[11] M. Bove, M. Grattarola, S. Martinoia, and G. Verreschi, "Interfacing cultured neurons to planar substrate microelectrodes: characterization of the neuron-to-microelectrode junction," Bioelect. Chem., Bioeng., vol. 38, pp. 255-265, 1995.

[12] L. J. Breckenridge, R. J. Wilson, P. Connolly, A. S. Curtis, J. A. Dow, S. E. Blackshaw, and C. D. Wilkinson, "Advantages of using microfabricated extracellular electrodes for in vitro neuronal recording," J. Neurosc. Res., vol. 42, pp. 266-276, 1995.

[13] R. Weis and P. Fromherz, "Frequency dependent signal transfer in neuron transistors," Phys. Rev. E., vol. 55, pp. 877-889, 1997.

[14] P. Fromherz, "Interfacing neurons and silicon by electrical induction," Ber. Bunsenges. Phys. Chem., vol. 100, pp. 1093-1102, 1996.

[15] S. Vassanelli and P. Fromherz, "Tranistor records of excitable neurons from rat brain," Appl. Phys. A., vol. 66, pp. 459-463, 1998.

[16] _ _ "Transistor probes local potassium conductances in the adhesion region of cultured rat hippocampal neurons," J. Neurosc., vol. 19, pp. 6767-6773, 1999.

[17] Biological Handbooks of the Federation of the American Societies for Experimental Biology: Growth. Bethesda, MD: Federation of the American Societies for Experimental Biology, 1962.

[18] Biological Handbooks of the Federation of the American Societies for Experimental Biology: Biological Databooks I and II. Bethesda, MD: Federation of the American Societies for Experimental Biology, 1974.

[19] T. H. Ziehen, Bardenleben's Handbuch des Anatomie IV 1-2, Das Nervensystem. Jena, Germany: Gustav Fischer Verlag, 1903

[20] J. R. Buitenweg, W. L. Rutten, W. P. Willems, and J. W. van Nieuwkasteele, "Measurement of sealing resistance of cell-electrode interfaces in neuronal cultures using impedance spectroscopy," Med. Biol. Eng. Comput., vol. 36, pp. 630-637, 1998.

[21] D. Braun and P. Fromherz, "Fluorescence interferometry of neuronal cell adhesion on microstructured silicon," Phys. Rev. Lett., vol. 81, pp. $5241-5244,1998$

[22] D. Gingel, "Cell contact with solid surfaces," in Biophysics of the Cell Surface, R. Glaser and D. Gingell, Eds. New York: Springer-Verlag, 1990, pp. 263-286

[23] A. Marty and E. Neher, "Tight seal whole cell recording," in Single Channel Recording, B. Sakmann and E. Neher, Eds. New York: Plenum, 1983, pp. 107-122.
[24] J. R. Buitenweg, W. L. C. Rutten, and E. Marani, "Modeled channel distributions explain extracellular recordings from cultured neurons sealed to microelectrodes," IEEE Trans. Biomed. Eng., vol. 49, Nov. 2002.

[25] — "Extracellular stimulation window explained by a geometry-based model of the neuron-electrode contact," IEEE Trans. Biomed. Eng., vol. 49, Dec. 2002.

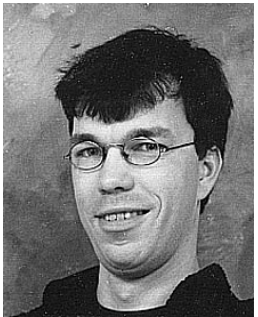

Jan Reinoud Buitenweg received the M.Sc. degree in biomedical engineering from the University of Twente, Enschede, The Netherlands, in 1998. He received the Ph.D. degree from the same institution in 2001 after studying the electrical behavior of the interface between cultured neurons and substrate embedded microelectrodes.

At present, he is an Assistant Professor with the University of Twente. His research interests are in information exchange with the neuromuscular system.

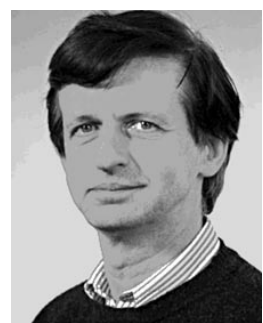

Wim L. C. Rutten received the Ph.D. degree in experimental physics from Leiden University, Leiden, The Netherlands, in 1979. Thereafter, he studied the auditory system at the ENT Department of Leiden University Hospital.

Since 1985, he has been with the BMTI/Faculty of Electrical Engineering of the University of Twente, Enschede, The Netherlands, as Assistant and Associate Professor of Biomedical Control Systems. His present research interests are neurotechnology (neuro-electronic interfaces, cultured probes), signal processing and bioelectricity.

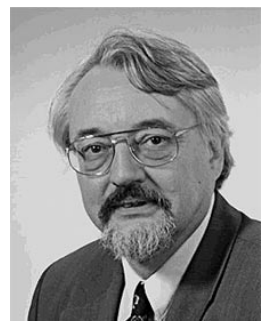

Enrico Marani received the Ph.D. degree in neuroanatomy from Leiden University, Leiden, The Netherlands, in 1982.

Thereafter, he became Head of the Neuroregulation group, Department of Physiology, Leiden University. Since 1997, he is part-time Professor in Neurophysiology at the BMTI / Faculty of Electrical Engineering of the University of Twente. His present research interests are neuroregulation and neurotechnology, especially in the field of nerve regeneration 